\title{
Enhancement of coherent Smith-Purcell radiation at terahertz frequency by optimized grating, prebunched beams, and open cavity
}

\author{
Peng Zhang, ${ }^{1, *}$ L. K. Ang, ${ }^{2}$ and A. Gover ${ }^{3}$ \\ ${ }^{1}$ Department of Nuclear Engineering and Radiological Sciences, University of Michigan, \\ Ann Arbor, Michigan 48109-2104, USA \\ ${ }^{2}$ Engineering Product Development, Singapore University of Technology and Design, \\ Singapore 138682, Singapore \\ ${ }^{3}$ Department of Electrical Engineering Physical Electronics, Tel Aviv University, Tel Aviv 69978, Israel
}

(Received 21 November 2014; published 26 February 2015)

\begin{abstract}
This paper studies various mechanisms to enhance the coherent Smith-Purcell radiation (SPR) at terahertz frequencies using particle-in-cell (PIC) simulations. A simple analytical dispersion relation is derived to predict the frequency of the evanescent surface wave of the open grating, which is excited by a backward-wave oscillation mechanism and emits radiation at harmonics of this frequency, and the cavity mode frequencies of the Orotron-like device, all in excellent agreement with the simulation data. The grating parameters are optimized to minimize the starting current of a continuous electron beam for exciting coherent SPR. It is demonstrated that a prebunched beam can be used to selectively excite coherent SPR at desired SPR angle and frequencies that are higher harmonics of the beam bunching frequency, where the starting current requirement is eliminated. Our PIC simulations show the dynamics of modes development and competition for the Orotron-like open cavity structure, which owns a lower starting current than the surface mode process. This oscillation mode dominates at steady state and enhances the radiated power by more than 2 orders of magnitude.
\end{abstract}

DOI: 10.1103/PhysRevSTAB.18.020702

PACS numbers: 41.60.Cr, 41.75.Ht

\section{INTRODUCTION}

Development of compact, tunable and high-power terahertz $(\mathrm{THz})$ sources has been drawing continuous interest in recent years [1-3]. Smith-Purcell radiation (SPR) is one particularly promising choice for developing such $\mathrm{THz}$ sources, which have significant potential applications in many areas, such as high-resolution imaging, noninvasive sensing, high-data rate communications and material analysis [1,2,4-11].

When electrons propagate close to the surface of metallic periodic grating, SPR could be emitted. It was first demonstrated in 1953 by Smith and Purcell [12], who used a $300 \mathrm{keV}$ electron beam to observe spontaneous radiation at visible wavelength. The radiation is governed by the SPR equation

$$
\lambda=\frac{L}{n}\left(\frac{1}{\beta}-\cos \theta\right) .
$$

Here $\lambda$ denotes the wavelength of the radiation produced at an angle $\theta$ with respect to the beam, $L$ is the grating period, $n$ is an integer, and $\beta=v / c$, where $v$ is the electron's

*umpeng@umich.edu

Published by the American Physical Society under the terms of the Creative Commons Attribution 3.0 License. Further distribution of this work must maintain attribution to the author(s) and the published article's title, journal citation, and DOI. velocity and $c$ is the speed of light. Incoherent SPR was studied in detail by many authors [13-16]. The idea of developing free electron lasers (FELs) based on coherent SPR was also proposed and analyzed [16-19]. In 1998, superradiant SPR was observed experimentally at Dartmouth College [20]. It was achieved due to the appearance of periodic electron bunching, induced by strong nonlinear interaction of the electron beam with an oscillating-evanescent wave (or grating surface mode) that is excited on the grating structure. The physics of coherent SPR has been studied extensively [21-26]. Many simulation works have been performed to investigate coherent SPR from continuously injected electron beam [27-30]. Several methods were suggested to enhance the SPR and to reduce the starting current, including the enhancement from surface plasmon excited by counterstreaming electron beams [31,32] and by adding sidewall to the grating [33]. In this paper, by using particle-in-cell (PIC) simulations, we identify the optimized grating parameters for minimizing the threshold (starting) current for coherent SPR. Contrary to previous simulation work $[28,34]$, we present the frequency spectrum of both the radiated waves and the beam current, which helps to directly verify the model of Andews et al. [22]. A simple analytical dispersion relation is derived for the open grating Smith-Purcell structure, which accurately predicts the operating frequency of the (evanescent surface mode) backward wave oscillation and the consequent coherent (superradiant) harmonic SPR. 
Since coherent SPR is achieved via beam bunching, it is essential to study the properties of radiation from a prebunched electron beam, which isolates the problem from the complicated evanescent mode beam bunching process. Tight prebunching in the form of a train of electron bunches can be produced from sources such as laserexcited photocathode [35], which can also be used to excite SPR. The radiation from the prebunched electron beams has been discussed thoroughly by Gover $[36,37]$ and Andrews et al. [23]. If the beam's pulse length of a single bunch is shorter than the SPR wavelength, enhanced coherent emission of phase correlated wave packets emitted in phase by the electrons of the bunch takes place (superradiant emission). When the electrons appear in periodic bunches, the coherent radiation from the bunches train interfere constructively, in directions where the frequency of the SPR is an integer multiple of the bunch repetition frequency, and significantly enhance the SPR emission in these directions. In 2005, Korbly et al. demonstrated such coherent harmonic SPR from a train of prebunched electrons experimentally [38]. Another distinct feature of the SPR excitation from prebunched electron beams is that the frequency and the direction of the radiation can be controlled by the beam bunching frequency, even for a given beam energy and fixed grating parameters, in contrast to the SPR excitation from a continuous beam, where the radiation is emitted in all directions in a continuous range of angle-dependent frequencies. We study in detail the effects of bunch number, beam current, and frequency selection on SPR.

In the open grating surface-wave-excited SPR device of Dartmouth College, the continuous electron beam is bunched at the evanescent frequency, which varies as the grating parameters changes, even for fixed beam energy and grating period [26], and thus limits the performance of such a device. By adding a metallic roof mirror directly above the grating (with a distance from the grating of integer multiples of half the SPR wavelength at $\theta=90^{\circ}$ ), an open cavity resonator is formed, similar to the Orotron [4,9,39-42]. The strong standing wave in the cavity interacts with the electron beam to cause bunching of the electrons. The bunch repetition frequency will be the same as the wave radiation frequency, which may give a larger coherence enhancement factor [23]. It should be emphasized that very extensive work has been done in the past on Orotrons, for example in Refs. [39,40]. However, our PIC simulations enable comparative study of the dynamics of the two different oscillation schemes that may develop and compete in the same structure: surface evanescent mode (with second harmonic SPR), and the cavity mode with an ideal Fabry-Perot resonator. The simulated operating frequency is accurately predicted by the analytical dispersion relation derived for the Orotronlike device.
In this study, we perform (2D) PIC simulations for coherent SPR, using the MAGIC code [43]. In Sec. II, the PIC simulation model is described. The results are presented in Sec. III. Simple, accurate analytical dispersion is derived to determine the oscillation condition for both the surface wave of the open grating and the cavity mode of the Orotron-like device, following which the simulation results are presented. We first study the open grating system with continuous electron beam. Incoherent and coherent SPRs are compared. The parameters of the grating are optimized to minimize the starting current for achieving coherent SPR. The results are compared to the theoretical analysis of Andrews et al. [22] and Kim et al. [26]. Next, we investigate the coherent SPR from prebunched electron beams. We examine the effects of bunch number, beam current, and frequency selection on SPR. The results are compared favorably with Gover's theory [36]. Finally, the coherent radiation from the Orotron-like device is studied for various injection currents. Salient radiation power enhancement is demonstrated. Conclusion is given in Sec. IV.

\section{THE SIMULATION MODEL}

Our simulation model is shown in Fig. 1. The perfect conductor rectangular grating is located at the bottom of the simulation box. We use a sheet electron beam with a thickness of $10 \mu \mathrm{m}$. The distance between the beam's bottom edge and the top of the grating is $10 \mu \mathrm{m}$. The beam is emitted uniformly from a cathode on the left near the grating and is a perfectly laminar flow. The injected beam current has a unit of $\mathrm{A} / \mathrm{m}$, where the length unit belongs to the $z$ direction (Fig. 1). The simulation box is enclosed by the freespace boundaries (or absorbers), which eliminate the reflection of the radiation from the

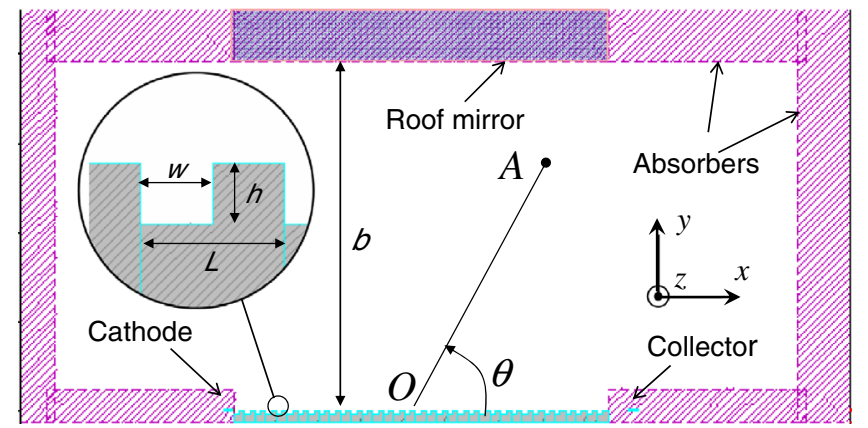

FIG. 1. MAGIC simulation model for SPR. The simulation box is enclosed by freespace boundaries (or absorbers). Perfect conducting rectangular grating is located at the bottom, directly above which there is a roof mirror area located on the upper boundary, which is set to freespace boundary for typical opengrating SPR simulation, and to perfect conductor for the Orotronlike simulation. Electron beam is emitted from the cathode, and absorbed at the collector after interaction with the grating. 
TABLE I. Main parameters used in simulation.

Beam energy

Beam thickness

Beam-grating distance

Roof-grating distance $b$

Grating period $L$

Grating groove height $h$

Grating groove width $w$

Number of grating periods

walls. There is a roof area located at the top boundary of the simulation box, direct above the grating. In the open grating SPR simulation, the roof area is set as a freespace boundary; whereas in the Orotron-like simulation, it is set as a perfect conductor, which would reflect back the radiation from beam-grating interaction and form an open cavity resonator with the grating. The whole simulation area is divided into meshes with small rectangular cells $(21 \mu \mathrm{m} \times 10 \mu \mathrm{m})$ in the region of beam propagation and grating, and big cells $(21 \mu \mathrm{m} \times 100 \mu \mathrm{m})$ in the rest. We summarize the main simulation parameters in Table I, which are used throughout the paper, unless otherwise stated. An infinite external magnetic field along the $x$ direction is used to ensure stable beam propagation above the grating.

\section{RESULTS}

\section{A. The surface wave and the Orotron-like oscillation conditions}

Before presenting the simulation results, we discuss the oscillation conditions for the open grating surface wave mode and the Orotron-like oscillation mode. For the open grating (Fig. 1 without roof), the dispersion equation is found to be

$$
\frac{\cot (\bar{\omega} \bar{H})}{\bar{\omega} \bar{H}}-\sum_{n=-\infty}^{\infty}\left(\frac{\sin \theta_{n}}{\theta_{n}}\right)^{2} \frac{\bar{W}}{\gamma_{n} \bar{H}}=0
$$

where $\quad \bar{\omega}=\omega L / c, \quad \gamma_{n}=\sqrt{p_{n}^{2}-\bar{\omega}^{2}}, \quad \theta_{n}=p_{n} \bar{W} / 2$, $p_{n}=\bar{k}+2 n \pi, \bar{k}=k L, \bar{W}=w / L, \bar{H}=h / L$, and $w, h$, $L$ are the width, height, and period of the grating, respectively (cf. inset of Fig. 1). Equation (2) is derived by assuming that the electromagnetic fields inside the grooves may be approximated by a TEM mode [10,44], which are then matched to the Floquet fields outside the grating, which behave like $e^{-\gamma_{n} y / L} e^{i\left(p_{n} x / L-\omega t\right)}$. The Floquet fields of all space harmonics are assumed to decay to zero at $y \rightarrow \infty$, thus, Eq. (2) is a valid solution only for the evanescent (surface mode) wave. Note that Eq. (2) resembles the cold-tube dispersion equation of a typical slow wave structure (SWS) [e.g., Eq. (77) of Ref. [44] and Eq. (8) of Ref. [10]]. In fact, in the limit of $\bar{W} \rightarrow 1$ in Eq. (2) and $b \rightarrow \infty$ in Eq. (77) of Ref. [44] and Eq. (8) of Ref. [10], these three equations become identical. Note also that Eq. (2) becomes identical to Eq. (33) of Andrews and Brau [22], when setting $m=n=0$ there. The open grating dispersion curve, Eq. (2), is plotted in Fig. 2, for the grating parameters given in Table I. It is clear that the entire curve for the surface wave mode is below the light line, which indicates that this surface wave mode is not radiating but evanescent in nature. The radiating Smith-Purcell wave is at the second harmonic of the evanescent surface wave.

When an electron beam propagates above the grating, the oscillation frequency of the grating surface wave (evanescent wave) that would be excited, $f_{e v}$ is determined by the intersection of the beam line and the dispersion relation of the open grating $\omega(k)$, that is the numerical solution of the dispersion equation, Eq. (2), as shown in Fig. 2. For an electron beam of $50 \mathrm{keV}$ and the grating parameters given in Table I, Eq. (2) gives $f_{e v}=0.717 \mathrm{THz}$, which is in excellent agreement with the simulation value of $0.71 \mathrm{THz}$ [cf. Figs. 5(d) and 5(f)], despite the fact that Eq. (2) is derived by assuming infinitely long grating, while the simulation was performed with a grating of 35 periods. It is also shown that the group velocity (the slope of the curve $d \bar{\omega} / d \bar{k})$ at the oscillation point is negative, indicating that the beam interacts with the backward wave. The excellent agreement of the oscillating frequency between the theory and the simulation verifies that the feedback mechanism (required for oscillation) is backward-wave oscillation (BWO), and not, for example, reflections from the edges of the grating. The BWO oscillation thresholds are studied in Refs. [45-48].

When there is a roof mirror added above the grating, a Fabry-Perot cavity between the mirror and the grating is expected to form, so that the device operates like an

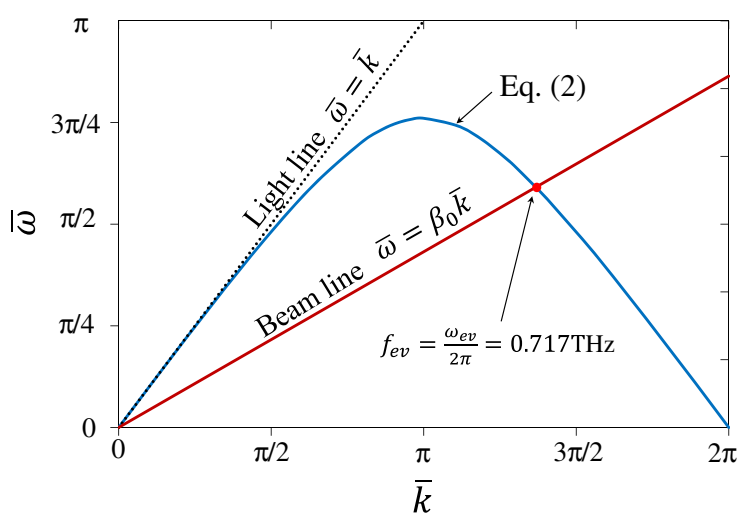

FIG. 2. The dispersion curve (blue), Eq. (2), of the open grating in Fig. 1, for the grating parameters given in Table I. The intersection point between the beam line $\bar{\omega}=\beta_{0} \bar{k}$ (red) and the dispersion curve gives the operating frequency of $f_{e v}=0.717 \mathrm{THz}$, for $50 \mathrm{keV}$ beam (ratio of beam velocity to speed of light $\left.\beta_{0}=0.4126\right)$. The dotted line is the light line $\bar{\omega}=\bar{k}$. 
Orotron (Fig. 1, with roof set to perfect conductor) [49]. The Orotron-like device resembles SWS [10,44] but with large roof-grating distance $b$. Similar to the open grating, the dispersion relation for the Orotron-like device is found to be

$$
\frac{\cot (\bar{\omega} \bar{H})}{\bar{\omega} \bar{H}}-\sum_{n=-\infty}^{\infty}\left(\frac{\sin \theta_{n}}{\theta_{n}}\right)^{2} \frac{\bar{W} \operatorname{coth} \gamma_{n} \bar{b}}{\gamma_{n} \bar{H}}=0
$$

where $\bar{b}=b / L$ is the normalized roof-grating distance, and all the other symbols have the same definitions as in Eq. (2). Note the only extra term $\operatorname{coth} \gamma_{n} \bar{b}$ is introduced in Eq. (3) as compared to Eq. (2). In the case of a surface wave mode all the space harmonics are evanescent, namely $\gamma_{n}$ is real for all $n(-\infty<n<\infty)$. In this case Eq. (3) is only a modification of Eq. (2) due to the boundary condition of the roof. In this case when $\bar{b} \rightarrow \infty$, Eq. (3) recovers Eq. (2), as expected. Note again the similarity between Eq. (3) and the cold-tube dispersion of SWS [e.g., Eq. (77) of Ref. [44] and Eq. (8) of Ref. [10]].

In the case of the Orotron all space harmonics are evanescent $\left(\gamma_{n}\right.$ is real) except $n=-1$, for which $\gamma_{n}=$ $i k_{y}$ is pure imaginary and radiating. In this case, a numerical solution of the dispersion equation (3) produces numerous dispersion curves that correspond in essence to the transverse TM modes of the parallel-plates waveguide, composed of the grating and the "Roof." These transverse mode dispersion curves are plotted in Fig. 3 for the parameters given in Table I. Inspection of Eq. (3) reveals that for $\bar{b} \gg 1(b \gg L)$ the evanescent harmonic terms in

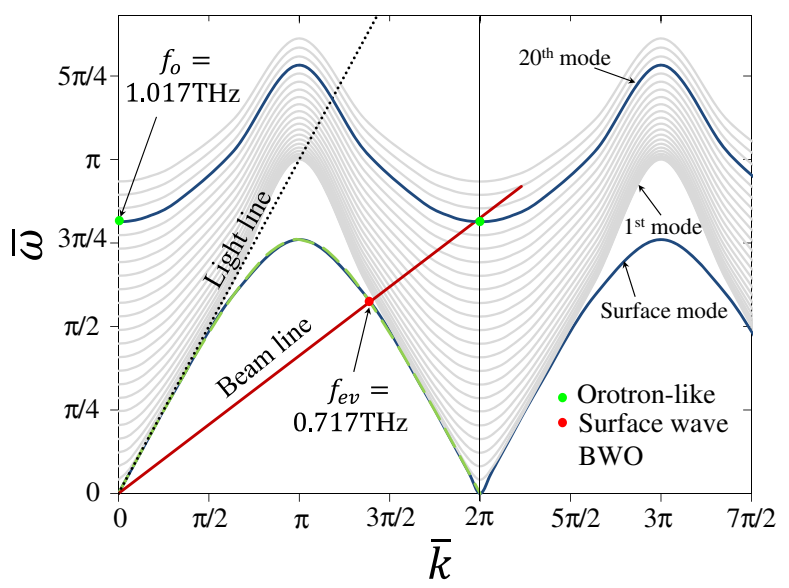

FIG. 3. The dispersion curves (blue and gray), Eq. (3), of the Orotron-like device in Fig. 1, for the grating parameters given in Table I. The beam line $\bar{\omega}=\beta_{0} \bar{k}$ (red) intersects with the surface mode at $f_{e v}=0.717 \mathrm{THz}$ (Smith-Purcell surface mode), and with the 20th mode close to its band edge at $f_{o}=1.017 \mathrm{THz}$ (Orotron-like cavity mode), for $50 \mathrm{keV}$ beam $\left(\beta_{0}=0.4126\right)$. The dotted line is the light line $\bar{\omega}=\bar{k}$, and the green dashed curve is the dispersion curve in Fig. 2, which is almost identical to the surface mode curve. the equation are negligible relative to the radiative space harmonic $n=-1$ term which is resonantly dominant for

$$
k_{y} b=m \pi(m=1,2, \ldots) .
$$

This space harmonic radiates vertically to the grating when $p_{-1}=0$, in which case $k_{y}=\omega=m \pi / b$, which corresponds to the Fabry-Perot resonant mode condition: $b=m \lambda / 2$. Since $\bar{b} \gg 1$ the spacing between the adjacent curves is small. For the parameters of Table I, $\Delta \omega=\pi / b=0.13$.

The dispersion curve of the surface wave mode (bottom blue curve in Fig. 3) is almost unaffected by the presence of the roof mirror at large distance $\bar{b}$, and is almost identical to the surface wave mode of the open grating (Fig. 2, reproduced as the green dashed curve in Fig. 3).

The beam line intersects with the dispersion curves of numerous transverse modes of the parallel plates waveguide. Besides the intersection with the surface wave mode (which gives the surface mode BWO oscillation frequency $f_{e v}=0.717 \mathrm{THz}$ ), the beam line also intersects with the dispersion curves of many other modes, and particularly with the $m=20$ mode of the Orotron resonator, near its band edge at $1.017 \mathrm{THz}$. It is known that band edge oscillation can be easily triggered by instability $[47,49,50]$, where the wave group velocity $d \bar{\omega} / d \bar{k}=0$. This means that a standing wave may be excited, which gives the Orotronlike resonant cavity mode at $f_{o}=1.017 \mathrm{THz}$, as shown in Fig. 3. Again, this is in excellent agreement with the e-beam interaction simulation value of $1.01 \mathrm{THz}$

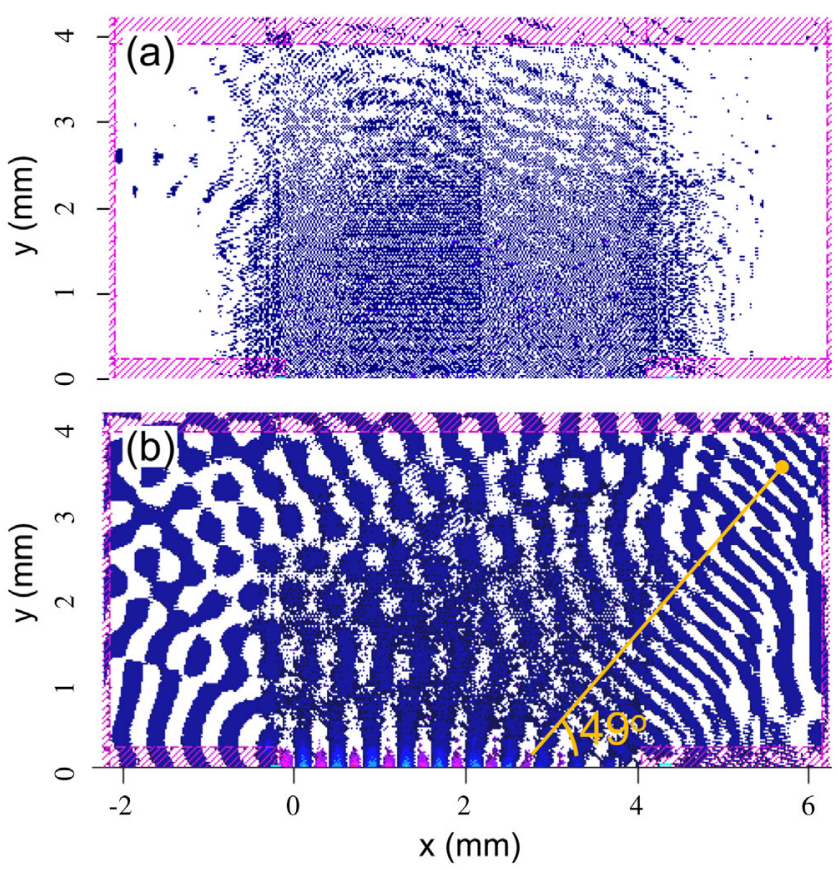

FIG. 4. Contour maps of magnetic field $B_{z}$ for a continuously injected electron beam of (a) $1000 \mathrm{~A} / \mathrm{m}$, and (b) $5000 \mathrm{~A} / \mathrm{m}$. Both of them are snapshots at $3 \mathrm{~ns}$ after the beam injection. 
[cf. Figs. 12(b), 14(d), and 14(e)]. As we shall see later from the simulation, the intersection points of the beam line with the other curves (1st-19th and $\geq 21$ st modes) are absent in the spectrum of the wave.

\section{B. Numerical simulation of continuous electron beam in an open structure}

When the injected current is small, the evanescent wave (or grating surface mode) is not strong enough to cause electron bunching. Thus, collective oscillation is not able to start, the radiation is spontaneous and incoherent. Figure 4(a) shows the contour map of the radiated magnetic field $B_{z}$ for an injection current of $1000 \mathrm{~A} / \mathrm{m}$. The radiation is not directional. The time history of $B_{z}$ (at location $A$ with $\theta=90^{\circ}$ and $O A=3 \mathrm{~mm}$ in Fig. 1) is given in Fig. 5(a) with its fast Fourier transform (FFT) spectrum in Fig. 5(b), showing a continuous frequency spectrum.

When the beam current exceeds a certain threshold value, called the starting current $I_{s}[25,26,45,46,49]$, the net gain of the signal can build up in time the BWO oscillation in the structure, and the beam gets bunched by the enhanced evanescent wave. For a given injection beam current density, we run the simulation and check whether the electron beam is bunched after 3 ns (which is about 100 times of the transit time for one electron to travel across the length of the whole grating). We gradually increase the injection current and repeat the simulation until the electron
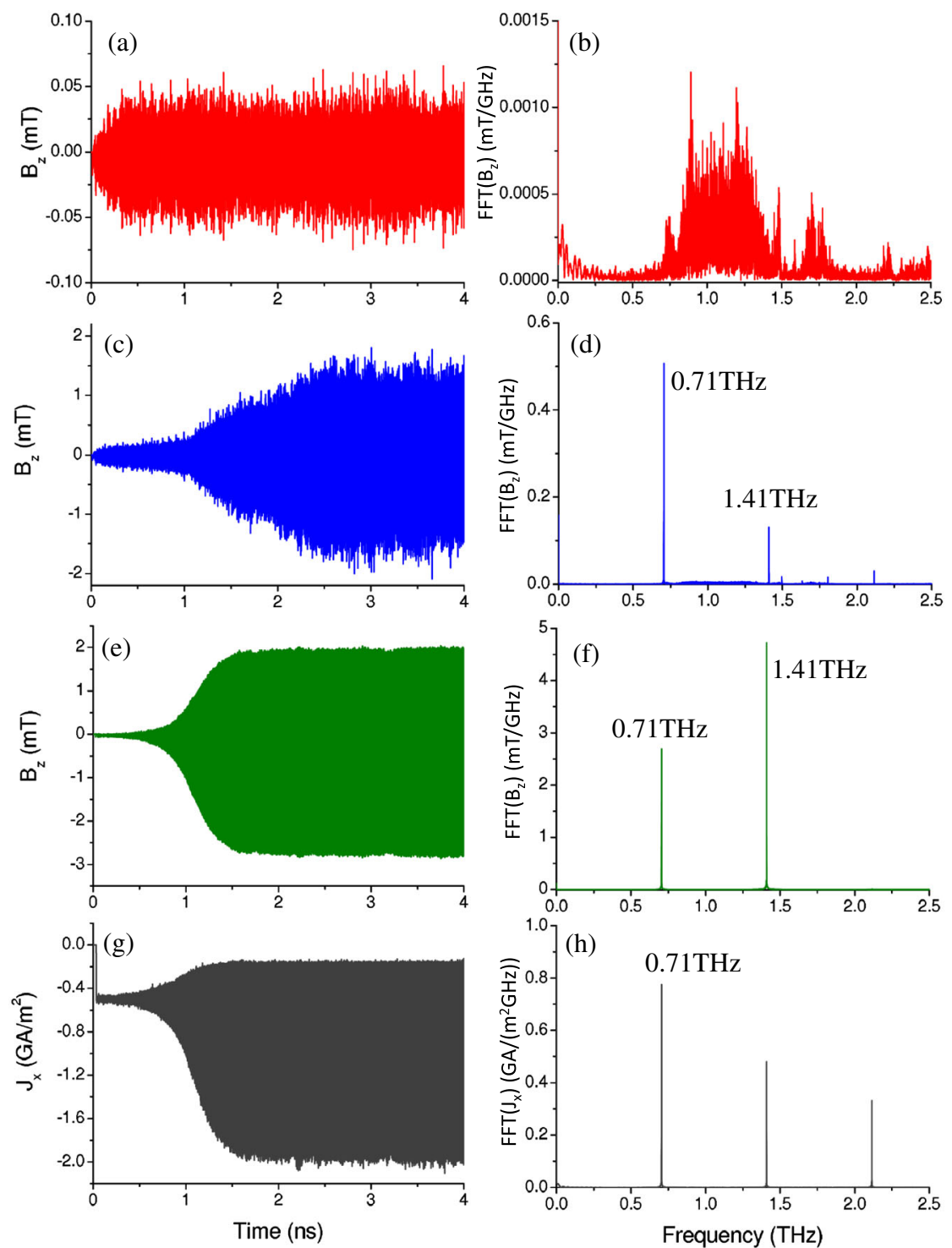

FIG. 5. Magnetic field $B_{z}(t)$ and its FFT detected at (a) and (b) $\theta=90^{\circ}$ with $O A=3.0 \mathrm{~mm}$ for a continuous beam of $1000 \mathrm{~A} / \mathrm{m}$, (c) and (d) $\theta=90^{\circ}$ with $O A=3.0 \mathrm{~mm}$ for continuous beam of $5000 \mathrm{~A} / \mathrm{m}$, (e) and (f) $\theta=49^{\circ}$ and $O A=4.5 \mathrm{~mm}$ for continuous beam of $5000 \mathrm{~A} / \mathrm{m}$. Also shown is the beam current density $(\mathrm{g}) J_{x}(t)$ and its FFT (h) detected at the middle point of the beam trajectory for $5000 \mathrm{~A} / \mathrm{m}$. 
beam bunching occurs. In this way, we found the starting current $I_{s} \approx 3000 \mathrm{~A} / \mathrm{m}$ for our device. For beam current density larger than the starting current, coherent SPR is excited with much larger intensity than the incoherent radiation. Figure 4(b) shows the $B_{z}$ contour map for an injection current of $5000 \mathrm{~A} / \mathrm{m}$. Besides the spherical wave radiation at the surface wave BWO frequency $f_{e v}=$ $0.71 \mathrm{THz}$ from both ends of the grating, there is a directional radiation of $1.41 \mathrm{THz}$ at a well-defined angle of $\theta=49^{\circ}$. This is the coherent SPR with frequency at the second harmonic of the BWO oscillation frequency of $2 f_{e v}(\cong 1.41 \mathrm{THz})$ ). It emits superradiantly at the angle $49^{\circ}$ predicted by the SPR equation, Eq. (1). The time history of $B_{z}$ (at $\theta=90^{\circ}$ with $O A=3 \mathrm{~mm}$ ) is shown in Fig. 5(c), where $B_{z}$ starts rapid growth around $1.0 \mathrm{~ns}$, with a saturated magnitude around $1.5 \mathrm{mT}$, much larger than that of incoherent radiation $[\sim 0.04 \mathrm{mT}$, cf. Fig. 5(a)]. Its FFT spectrum is given in Fig. 5(d), showing frequency peaks at both 0.71 and $1.41 \mathrm{THz}$. At $\theta=49^{\circ}$, the dominant frequency of the radiation is at $1.41 \mathrm{THz}$, as shown in Figs. 5(e) and 5(f). For infinitely long grating, the evanescent wave decays exponentially away from the top surface of the grating, which behaves like $B_{z} \sim e^{-\gamma_{n} y}$, where $\gamma_{n}$ is the decay constant [see the sentence following Eq. (2)]. Thus, the evanescent field penetration range would be $1 / \gamma_{n}$, which is found to be $11.4,30.46$, and $149 \mu \mathrm{m}$, for $n=-1,0,1$, respectively, much smaller than the vertical distance of the detection point from the grating $(3 \mathrm{~mm}$ for $\theta=90^{\circ}, 3.4 \mathrm{~mm}\left(=4.5 \mathrm{~mm} \times \sin 49^{\circ}\right)$ for $\left.\theta=49^{\circ}\right)$. Therefore, the detected field at $0.71 \mathrm{THz}$ is most likely due to the diffraction from the two ends of the grating, which is also evident from the contour map shown in Fig. 4(b). The beam current density $J_{x}(t)$ at the middle point of its trajectory is plotted in Fig. 5(g) with its FFT in Fig. 5(h). The fundamental beam bunching frequency is $0.71 \mathrm{THz}$, which is the same as the evanescent wave frequency $f_{e v}$. The higher harmonics of the bunching frequency are also presented in the spectrum.

It is desirable to minimize the starting current $I_{s}$ for obtaining coherent SPR. We examine the effects of the grating parameters (groove height $h$ and width $w$, as shown in Fig. 1) on $I_{s}$, with the grating period $L$ and injection beam energy fixed. Figure 6(a) shows the approximate starting current $I_{s}$ as a function of the groove height $h(w$ is kept at $60 \mu \mathrm{m}$ ) as well as groove width $w$ ( $h$ is kept at $100 \mu \mathrm{m}$ ), where all the other parameters remain the same as those in Table I in the simulation. A minimum starting current $I_{s}$ is found as low as $14( \pm 2) \mathrm{A} / \mathrm{m}$, for $h=100 \mu \mathrm{m}$ and $w=60 \mu \mathrm{m}$, where $I_{s}$ is reduced by a factor of more than 200 from that with grating parameters in Table I. It is important to note that though the grating period $L$ and electron beam energy are fixed, the evanescent wave frequency $f_{e v}$ (or the evanescent wavelength $\lambda_{e v}$ ) varies quite significantly with different $h$ and $w$, as shown in Fig. 6(b), so does the beam bunching frequency.
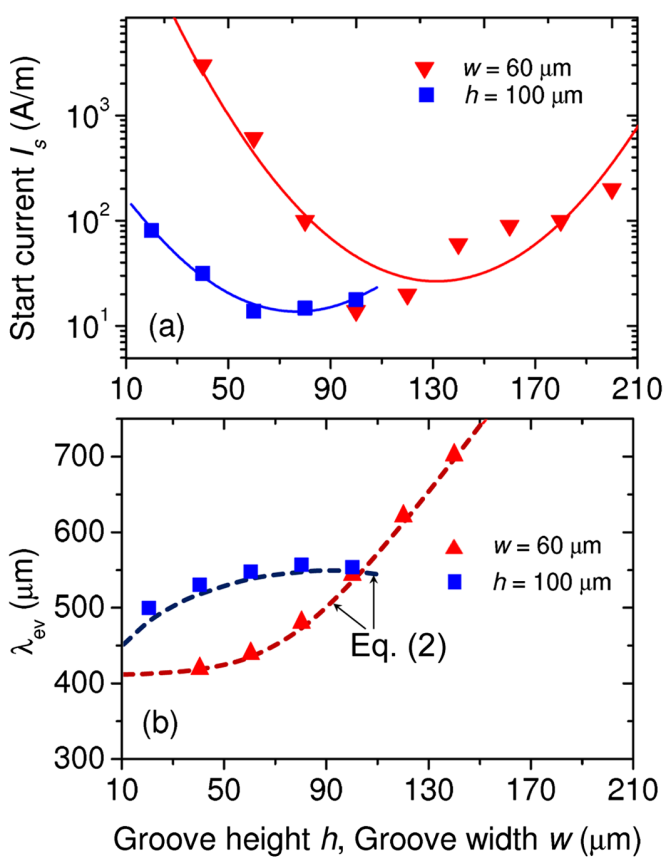

FIG. 6. (a) Starting current $I_{s}$ and (b) the corresponding evanescent wavelength $\lambda_{e v}$, as a function of the groove height $h$ (triangles, groove width $w$ fixed at $60 \mu \mathrm{m}$ ) as well as groove width $w$ (squares, groove height $h$ fixed at $100 \mu \mathrm{m}$ ). The other parameters are given in Table I. Solid lines in (a) are curve fittings to the simulation data, dashed lines in (b) are analytical calculation from Eq. (2). Note the very good agreement between the simulation and the theory.

Therefore, the SPR frequency (the second harmonic of $f_{e v}$ ) also changes with different $h$ and $w$. Note the very good agreement for $\lambda_{e v}$ obtained from the simulation (symbols) and the theory from Eq. (2) (dashed lines) in Fig. 6(b). Our results indicate that coherent SPR at $2 f_{e v}=$ $1.09 \mathrm{THz}\left(\lambda_{e v}=550 \mu \mathrm{m}\right)$ can be obtained for a grating of $h=100 \mu \mathrm{m}$ and $w=60 \mu \mathrm{m}$ operating at $I_{s} \approx$ $12-16 \mathrm{~A} / \mathrm{m}$. Note that the results for the starting current $I_{s}$ and the evanescent wavelength in Fig. 6 show similar trends to those of Kim and Kumar [51].

\section{Numerical simulation of a prebunched electron beam in an open structure}

A square-wave pulse of current (train of rectangularshaped electron bunches) of $1000 \mathrm{~A}$ is emitted from the cathode with a bunching repetition frequency $f_{b}=1.03 \mathrm{THz}$, which equals to the SP frequency at $\theta=$ $90^{\circ}$ for the parameters in Table I. The length of each electron bunch is half of the bunching period $\left(1 / 2 f_{b}=0.485 \mathrm{ps}\right)$. Figure 7 shows the time history of magnetic field component $B_{z}$ at $\theta=90^{\circ}$ along with their FFTs, for periodic trains of electron bunches with different number of bunches $N_{M}: N_{M}=6$ [(a) and (b)], $N_{M}=16$ [(c) and (d)], and $N_{M}>100$ [(e) and (f)]. It is clear that the radiation frequency at $1.03 \mathrm{THz}$ is the same as bunching 

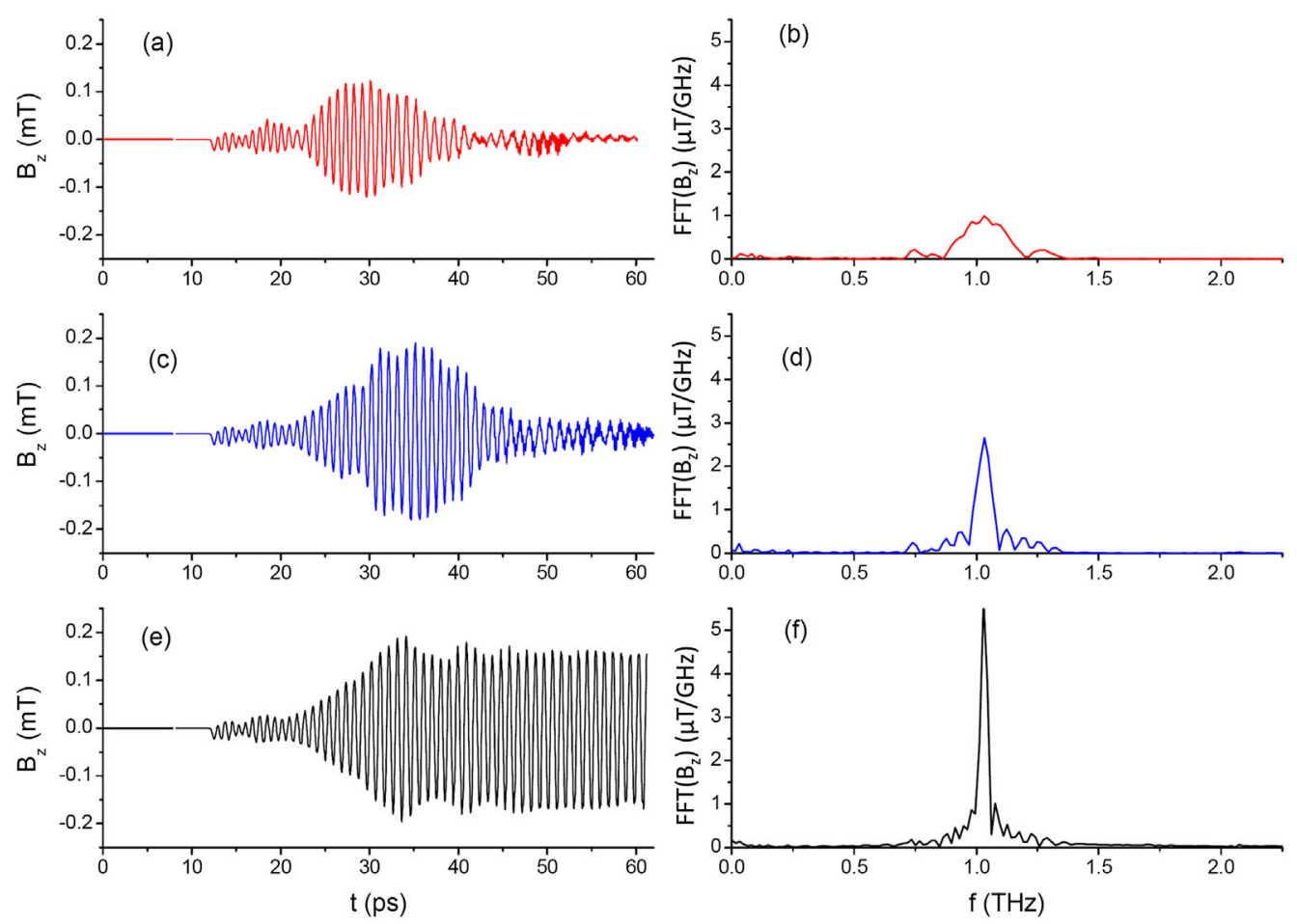

FIG. 7. Magnetic field $B_{z}$ (left) and its FFT (right) for SPR from periodic trains of different number of electron bunches $N_{M}$ : (a) and (b) for $N_{M}=6$; (c) and (d) for $N_{M}=16$; and (e) and (f) for $N_{M}>100$. The $B_{z}$ is detected at $\theta=90^{\circ}$ with $O A=3 \mathrm{~mm}$.

repetition frequency $f_{b}$. As the number of the electron bunches $N_{M}$ increases, the bandwidth near $f_{b}$ decreases, being inversely proportional to $N_{M}$, which agrees well with the theory prediction $[36,38]$. In particular, the spectral energy is found as

$$
\frac{d W}{d \omega} \propto\left[\frac{\sin \left(N_{M} \pi \omega / \omega_{b}\right)}{N_{M} \sin \left(\pi \omega / \omega_{b}\right)}\right]^{2},
$$

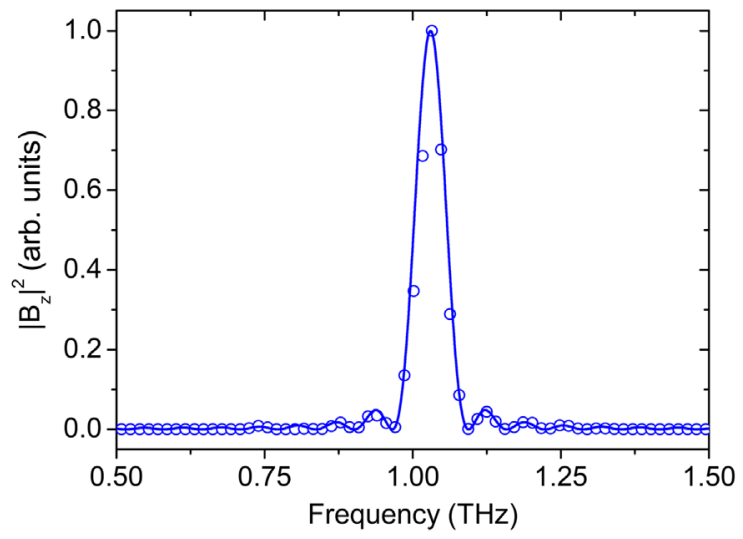

FIG. 8. Comparison of the square of FFT of $B_{z}$ (after normalization of the peak value to 1.0) (circle) and the theoretical prediction (solid line) for $N_{M}=16$. The peak corresponds to a radiation frequency of $1.03 \mathrm{THz}$ and has $60 \mathrm{GHz}$ FWHM. where $\omega_{b}=2 \pi f_{b}$. Figure 8 shows the normalized spectral energy ( $\alpha$ square of FFT of $B_{z}$ ), which is compared to the theoretical curve from Eq. (4), for the case $N_{M}=16$ [Fig. 7(d)]. Excellent agreement between the simulation and the theory is noted.

Since the bunch length is shorter than the radiation wavelength in our simulation, the radiation from the prebunched electron beam is always coherent, regardless of how small the injection current is. Thus, there is no starting current limitation for coherent SPR from periodic

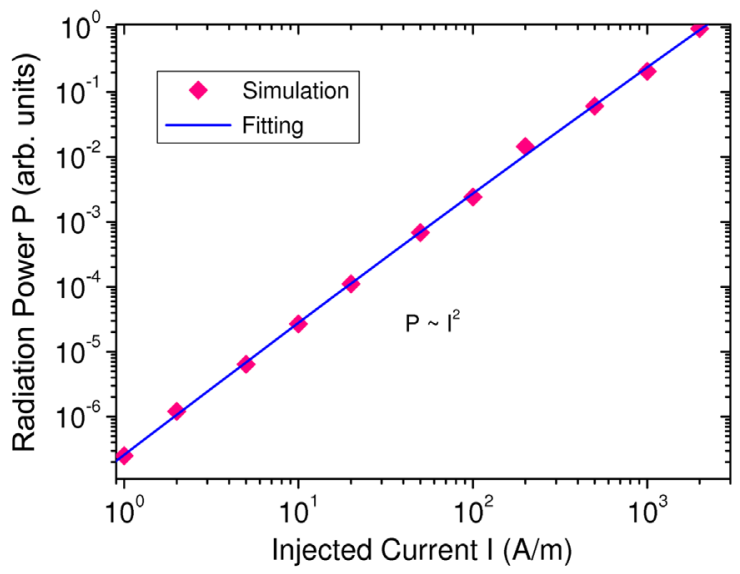

FIG. 9. Detected radiation power $P$ from prebunched electron beams as a function of the injection current $I$. 

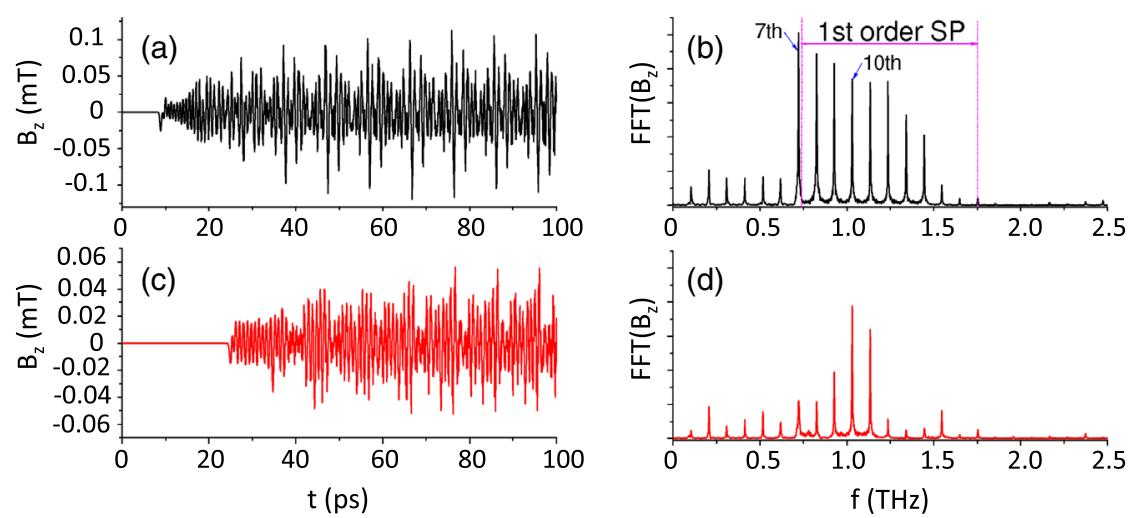

FIG. 10. Magnetic field at $\theta=90^{\circ}$ (a) detected at $O A=1.5 \mathrm{~mm}$ with (b) its FFT, and (c) detected at $O A=7 \mathrm{~mm}$ with (d) its FFT, excited from a prebunched beam of bunching frequency $f_{b}=0.103 \mathrm{THz}$, with the length of each bunch as 0.485 ps at $N_{M}>100$.

prebunched electron beam, as confirmed from our simulation. Figure 9 shows that the detected radiation power $P$ increases as the square of the beam current $I$, i.e., $P \propto I^{2}$. Since the number of electrons in each bunch $N_{b}$ is proportional to the beam current $I, N_{b} \propto I$, this simulated scaling agrees well with the theoretical analysis by Gover [36],

$$
P \propto N_{b}^{2}
$$

For common electron guns, it may be hard to attain electron bunch repetition frequency $f_{b}$ as high as in the $\mathrm{THz}$ regime. Therefore we examine the scheme of exciting $\mathrm{THz}$ radiation using a beam bunched at high subharmonic of the $\mathrm{THz}$ radiation frequency. We performed prebunched beam SPR simulation with a low bunching frequency $f_{b}=103 \mathrm{GHz}$, which is the tenth subharmonic of the SPR emission frequency $(1.03 \mathrm{THz})$ at $90^{\circ}$. The injected current is $1000 \mathrm{~A}$ and the bunching length is kept the same as the previous case of 0.485 ps. Figures 10 (a) and 10(b) show the magnetic field $B_{z}$ as a function of time $t$ and its FFT, respectively, which are detected $1.5 \mathrm{~mm}$ above the center of the grating. Though the FFT of the electron current shows the full range (1st-20th) of harmonics of the bunching frequency $f_{b}$, the major frequency components of the radiated waves lie only in the regime of first order SPR, governed by Eq. (1) with $n=1$. The seventh harmonic of $f_{b}$ is $0.721 \mathrm{THz}$, which is lower than the minimum allowed SP frequency of $0.73 \mathrm{THz}$ according to Eq. (1), is also presented in the spectrum. This frequency is very close to the evanescent wave frequency $f_{e v}=0.71 \mathrm{THz}$. When the detector is moved to $7 \mathrm{~mm}$ further from the center of the grating, the frequency components of $B_{z}$ are confined to an even smaller radiation zone near to the $90^{\circ} \mathrm{SPR}$ of $1.03 \mathrm{THz}$, as shown in Fig. 10(c) with its FFT in Fig. 10(d). This demonstrates that coherent SPR of frequency $f_{s p}$ can be selectively excited by a prebunched beam of subharmonic bunching frequency $f_{s p} / n$, where $n>1$ is an integer.

\section{Numerical simulation of an Orotron-like configuration}

In this section, the device configuration is similar to the previous cases, except that the roof mirror area is set as a perfect conductor, as shown in Fig. 1. The distance between the mirror and the grating is adjusted to integer multiples of half the wavelength of the $90^{\circ} \mathrm{SPR}$ of $1.03 \mathrm{THz}$ [as predicted from Eq. (1)], thus it is expected that a resonant Fabry-Perot kind of cavity is formed at this frequency, similar to the Orotron. A continuous electron beam is injected into the interaction region above the grating. Although electrons interact with both the standing wave cavity mode and the first space harmonic of the surface evanescent mode, for which the condition of the Cherenkov synchronism is fulfilled (Fig. 3), as we shall see in Figs. 11 and 12 that beam bunching is only produced via the interaction with the strong standing wave, for the given current level of $1000 \mathrm{~A} / \mathrm{m}$. In Fig. 11, the contour map of the radiated $B_{z}$ at $t=20 \mathrm{~ns}$ is displayed for current of $1000 \mathrm{~A} / \mathrm{m}$. A strong resonant oscillation is built up between the grating and the roof mirror. The time history of the radiated magnetic field $B_{z}$, detected directly above the center of the grating from a distance of $1.5 \mathrm{~mm}$ along with its FFT are shown in Figs. 12(a) and 12(b),

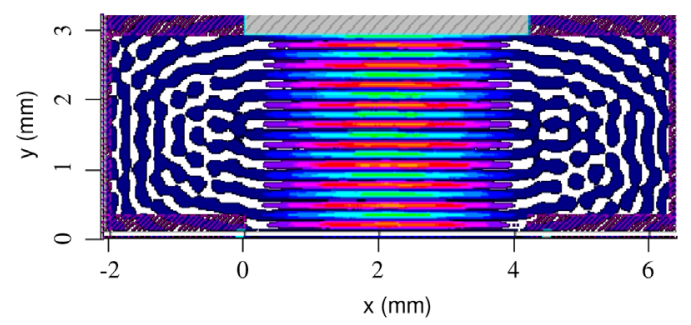

FIG. 11. Contour map of $B_{z}$ at $20 \mathrm{~ns}$ for continuously injected electron beam of $1000 \mathrm{~A} / \mathrm{m}$ in the Orotron-like configuration. 

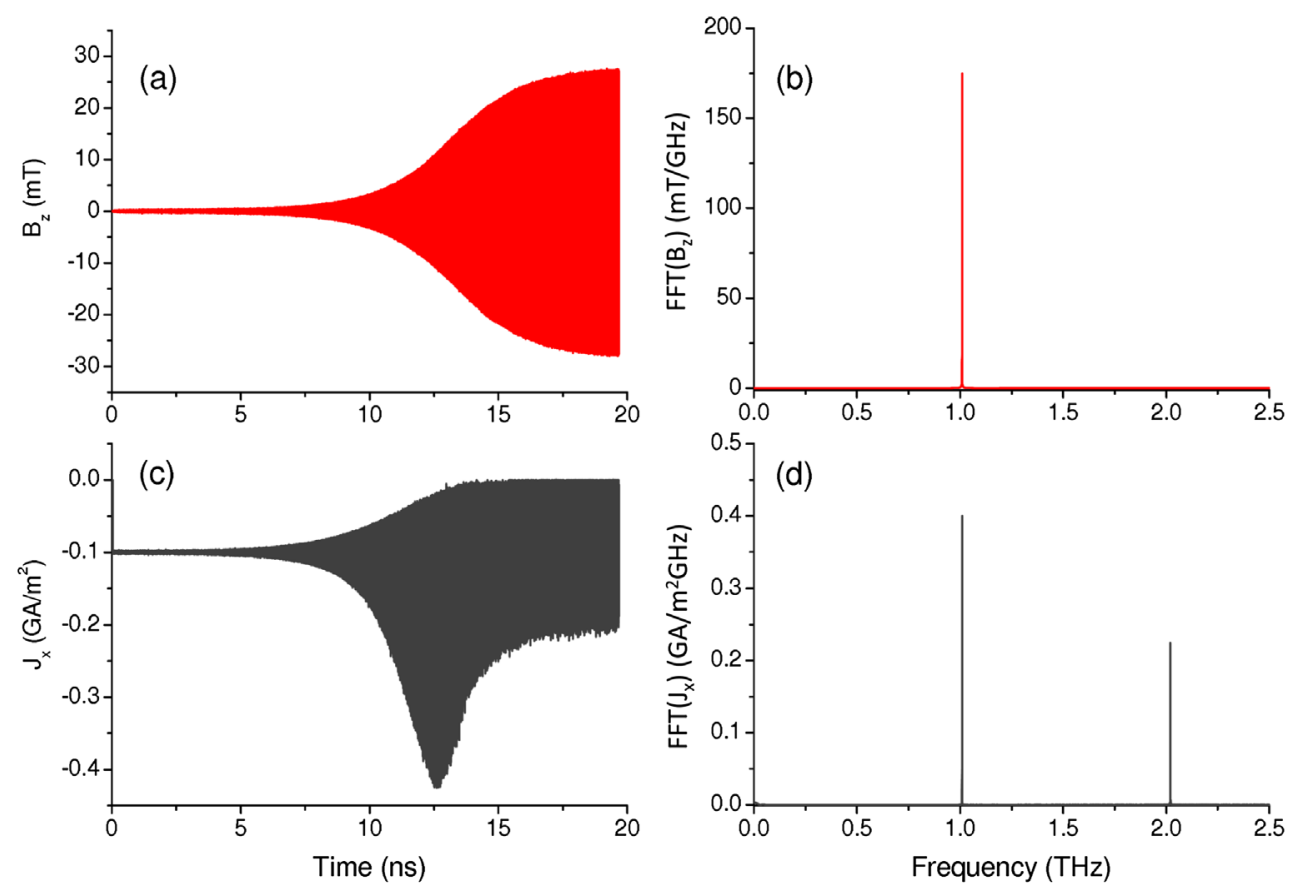

FIG. 12. (a) $B_{z}(t)$ at $\theta=90^{\circ}$ with $O A=1.5 \mathrm{~mm}$, (b) its FFT, (c) current density $J_{x}(t)$, and (d) its FFT, for continuously injected beam of $1000 \mathrm{~A} / \mathrm{m}$ in the Orotron-like configuration.

respectively. After $10 \mathrm{~ns}$, the field starts to grow rapidly and becomes saturated around $20 \mathrm{~ns}$, with a magnitude of nearly $30 \mathrm{mT}$, which is immensely larger than the $B_{z}$ from open grating with the same grating parameters, beam energy and injected current magnitude $[0.05 \mathrm{mT}$ for continuous beam, Fig. 5(a); $0.15 \mathrm{mT}$ for prebunched beam, Fig. 7(e)]. Its FFT indicates clearly that there is only single radiation frequency at $f_{o}=1.01 \mathrm{THz}$ in its spectrum. Figures 12(c) and 12(d) show the injection current density profile as a function of time and its FFT, respectively. It is obvious that the initially continuous electron beam is bunched at the same frequency as the radiated wave at $f_{o}=1.01 \mathrm{THz}$. Note the slight difference between the frequency of $1.01 \mathrm{THz}$ for the Orotron-like device, as compared to that of $1.03 \mathrm{THz}$ for the open grating device in Sec. III C. As predicted by the dispersion equation, Eq. (3), in Sec. III A, this oscillation frequency of $f_{o}=1.01 \mathrm{THz}$ is due to the band edge oscillation (Fig. (3)), even though at $\bar{k}=2 \pi$, the beam line gives $\bar{\omega}=\beta_{0} \bar{k}=2.592$, corresponding to $1.03 \mathrm{THz}$. Physically, this is due to the nature of the mirror-grating cavity, where the effective cavity size may not be exactly the same as the distance between the bottom of the mirror and the top of the grating, since the grating is not a flat surface. The grating may act as a mirror with phase shifts.

Similar to the open grating SPR in Sec. III B, the continuous injection current in the Orotron-like configuration must be larger than a threshold current (or the starting current) $I_{o}$, in order to build up the oscillation and to form beam bunching. In our simulation, we found
$I_{o} \approx 500 \mathrm{~A} / \mathrm{m}$, which is only one sixth of that for the open grating device $\left(I_{s} \approx 3000 \mathrm{~A} / \mathrm{m}\right)$, for the same grating parameters and beam energy in Table I. Thus, our PIC simulations confirm that the Orotron-like configuration may be used to reduce the starting current requirement for achieving coherent SPR for continuous electron beam [41]. It should be noted that the starting current in the Orotron-like configuration depends on the quality factor $Q$

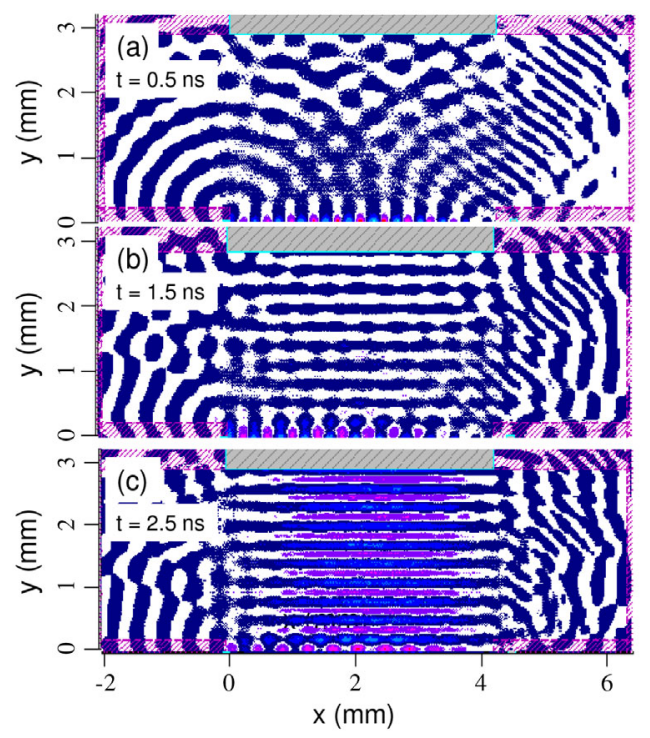

FIG. 13. Contour map of $B_{z}$ for continuously injected electron beam of $5000 \mathrm{~A} / \mathrm{m}$ in the Orotron-like device at (a) $0.5 \mathrm{~ns}$, (b) $1.5 \mathrm{~ns}$, (c) $2.5 \mathrm{~ns}$. 
of the open resonator $[39,40]$. For this theoretical study, we took the simplest case of $100 \%$ reflective top mirror, which corresponds to the minimal effect of $Q$-factor due to escape of radiative power to all harmonics and diffraction loss at the side ends of the resonator $[39,40]$.

When the injected current exceeds $I_{o}$ (the starting current for the Orotron-like device) but is smaller than $I_{s}$ (the starting current for the open grating device), the standing wave (Fabry-Perot mode) formed between the roof mirror and the grating is stronger than the evanescent surface mode of the grating. Thus, the electron beam is bunched by the standing wave [cf. Fig. 12(d)] and only the Orotron-like oscillation is excited, where the evanescent wave oscillation is absent [cf. Fig. 12(b)].

When the injected current exceeds $I_{s}$ (the starting current for the open grating device), the evanescent wave would become significant, which may drive the electron beam to bunch at frequency $f_{e v}$ temporarily. Figure 13 show the snapshots of $B_{z}$ contour map at different times for an injected current of $5000 \mathrm{~A} / \mathrm{m}\left(>I_{s}>I_{o}\right)$. At $0.5 \mathrm{~ns}$, it shows radiation from both ends of the grating and a directional radiation at angle $\theta=49^{\circ}$, similar to the open-grating coherent SPR in Fig. 4(b), which indicates that the electron beam is bunched at the evanescent frequency $f_{e v}=0.71 \mathrm{THz}$ and its second harmonic, and radiates at the second harmonic frequency $2 f_{e v}$. At $1.5 \mathrm{~ns}$, there are some standing wave patterns present between the grating and the roof mirror. But the directional radiation at $\theta=49^{\circ}$ is still presented. The electron beam interact simultaneously with both the standing wave of $f_{o}=$ $1.01 \mathrm{THz}$ and the evanescent wave of $f_{e v}=0.71 \mathrm{THz}$, both of which attempt to get the electron beam bunched at their specific frequencies in a typical mode competition process of an homogeneously broadened laser oscillator [52]. At $2.5 \mathrm{~ns}$, the standing wave in the contour map becomes dominant and its intensity becomes stronger.
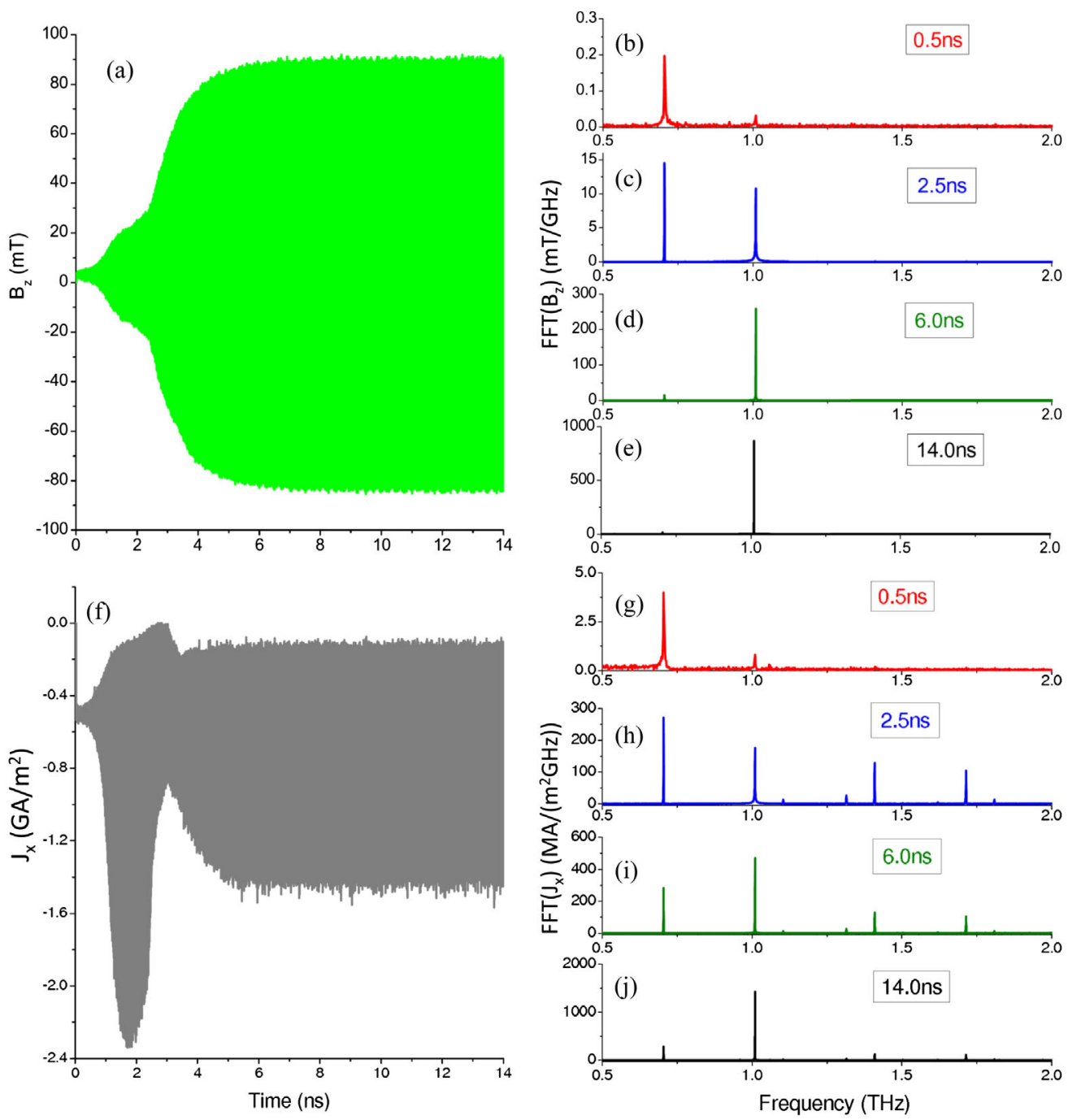

FIG. 14. (a) $B_{z}(t)$ in the grooves of the grating, and its FFT at (b) $0.5 \mathrm{ns,} \mathrm{(c)} 2.5 \mathrm{~ns}$, (d) $6.0 \mathrm{~ns}$, (e) $14.0 \mathrm{~ns}$; (f) current density $J_{x}(t)$ and its FFT at (g) $0.5 \mathrm{~ns}$, (h) $2.5 \mathrm{ns,} \mathrm{(i)} 6.0 \mathrm{~ns}$, (j) $14.0 \mathrm{~ns}$, for continuous injected beam current of $5000 \mathrm{~A} / \mathrm{m}$ in the Orotron-like device. 
At $6.0 \mathrm{~ns}$, the contour map become almost identical to that in Fig. 11, indicating that the electron beam is eventually bunched at the standing wave frequency $f_{o}=1.01 \mathrm{THz}$. The standing wave radiation becomes saturated and the system operates in the dominant "Orotron operating mode" at steady state.

The time history of $B_{z}$ detected in the grooves of the grating along with its FFTs at several different time are shown in Figs. 14(a)-14(e). Figures 14(f)-14(j) show the time history of the beam current bunching and its FFT at various time. At $0.5 \mathrm{~ns}$, the frequency component at $f_{e v}=$ $0.71 \mathrm{THz}$ for both the magnetic field $B_{z}$ and the beam current is much larger than that at $f_{o}=1.01 \mathrm{THz}$, which means the interaction between the electron beam and the evanescent wave is stronger and the beam is bunched mostly by the evanescent wave. At $2.5 \mathrm{~ns}$, the peak at $f_{o}=$ $1.01 \mathrm{THz}$ becomes comparable to that at $f_{e v}=0.71 \mathrm{THz}$, indicating that the interaction between the beam and the standing wave becomes significant, and the beam is modulated by both the evanescent wave and the standing wave. At $6.0 \mathrm{~ns}$, both $B_{z}$ and the current become saturated and the peak at $f_{o}$ is larger than that at $f_{e v}$. At $14 \mathrm{~ns}$, the peak at $f_{o}$ becomes almost 6 times larger than that at $f_{e v}$. The magnitude of $B_{z}$ under steady state is about $90 \mathrm{mT}$, compared to that of about $2.5 \mathrm{mT}$ in Fig. 5(e) for the open grating device. This indicates that the radiated power $\left(\propto B_{z}^{2}\right)$ is enhanced by more than 2 orders of magnitude by using the Orotron-like open cavity configuration.

As shown in Fig. 3, the beam line intersects with both the grating surface mode (i.e., the evanescent wave) curve and the resonant cavity mode (i.e., the standing wave at band edge). The magnitude of the injected current determines during the oscillation build-up period the relative strength of the evanescent wave and the standing wave at different times, both of which in turn could modulate the beam current density. Under steady state, as long as the injected current density is larger than the starting current $I_{o}$, the electron beam will be eventually bunched by the standing wave and the radiation is "pinned" at the cavity resonant

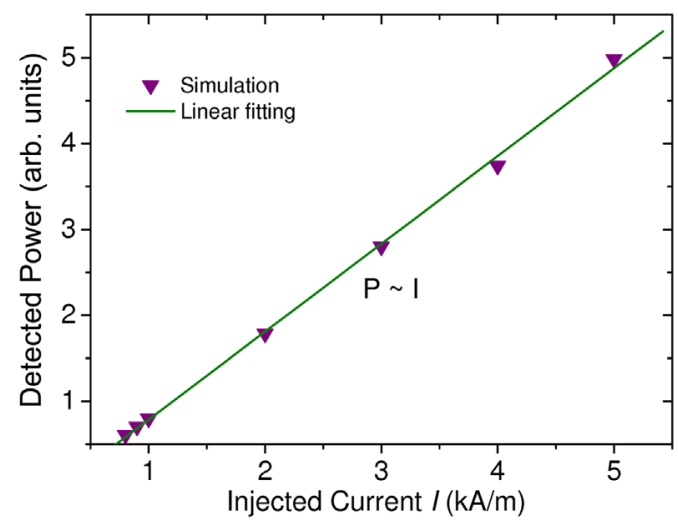

FIG. 15. Radiation power $P$ as a function of the injection current $I$ in the Orotron-like device. frequency (band edge oscillation) $f_{o}$. It is found that the power of the standing wave is linearly proportional to the injected current density for the Orotron-like configuration, as shown in Fig. 15.

\section{CONCLUDING REMARKS}

In summary, we have studied coherent SP radiation by 2D PIC simulation for both open grating configuration and Orotron-like configuration, driven by different modes of the injected electron beam. Simple analytical dispersion relations are derived for both the open grating and the Orotron-like device, which predict the oscillation conditions in excellent agreement with the simulated values. In the open grating device, coherent SP radiation is generated when the injected continuous beam current exceeds the starting current $I_{s}$ for backward-wave oscillation of the grating surface mode. The electron beam is bunched by the strong evanescent wave frequency $f_{e v}$ and its second harmonic component coherently radiates SP wave at $2 f_{e v}$ at the specific SP direction. The study included optimization of grating parameters to minimize $I_{s}$. We further demonstrated coherent radiation emission by a prebunched beam, in which the starting current requirement is eliminated. We showed that the radiated power from prebunched beams is proportional to the square of the injected current, as expected in superradiant emission processes. The Orotron-like configuration is verified to drastically lower the starting current requirement for the generation of coherent SP radiation, where the radiated power is linearly proportional to the injected current. The radiation power is enhanced by more than 2 orders of magnitude as compared to the typical open grating device.

We showed the operating mode competition process during oscillation build-up in the Orotron-like SP structure, in which the evanescent surface wave BWO and harmonic radiation mode is first excited and then the dominant standing-wave Orotron oscillation mode settles at saturation.

Last, we would like to address a few practical considerations in realizing the devices studied in this theoretical paper. In the PIC simulation, an infinite strong magnetic field (2T) is used to ensure stable propagation of the electron beam above the grating. In practice, due to the available magnetic field, issues such as inevitable beam halo, inclination and spread of transverse velocities have to be considered.

A careful reader may already notice the high injection current of $1000 \mathrm{~A} / \mathrm{m}$ and $5000 \mathrm{~A} / \mathrm{m}$ used in the simulations (e.g., Figs. 4, 5, 12-14). Even for the perfectly conducting structure, the starting current for the parameters in Table I is found to be as high as $3000 \mathrm{~A} / \mathrm{m}$, which could be very difficult to realize in practice. However, we should note that the grating parameters in Table I are not optimized, as already discussed in Sec. III B. These unrealistically high currents particularly indicate the significance of the optimization of grating parameters. The optimized grating $(h=100 \mu \mathrm{m}$ and $w=60 \mu \mathrm{m})$ would be 
able to produce coherent $\mathrm{THz}$ SPR at current $\approx 12-16 \mathrm{~A} / \mathrm{m}$ (Fig. 6), corresponding to $0.12-0.16 \mathrm{kA} / \mathrm{cm}^{2}$ (with a beam thickness of $10 \mu \mathrm{m}$ ), which is well below the available state-of-art beam current [53]. For the prebunched beam (Fig. 7) with a current of $I=1000 \mathrm{~A} / \mathrm{m}$ and duration of $\tau=0.485 \mathrm{ps}$, the total charge per bunch would be $q=I \tau=0.485 \mathrm{nC} / \mathrm{m}$, which is close to the realistic value [53], however, there is no starting current requirement for coherent SPR from prebunched beam, as discussed in Sec. III C.

For this theoretical study, we took the simplest case of $100 \%$ reflective top mirror, which corresponds to the minimal effect of $Q$-factor due to escape of radiative power to all harmonics and diffraction loss at the side ends of the resonator. The oscillation threshold in a real Orotron is expected to be higher [39-41], because the roof must transmit partly (or through a hole) in order to get radiation out, and because of Ohmic losses, as is known in the extensive work on Orotrons [39-41].

\section{ACKNOWLEDGMENTS}

P.Z. was supported in part by Air Force Office of Scientific Research (AFOSR) Grant No. FA9550-14-10309. L. K. A. would like to acknowledge the support the Massachusetts Institute of Technology-Singapore University of Technology and Design International Design Center (MIT-SUTD IDC) Grants (No. IDD21200103 and No. IDG21200106).

[1] P. H. Siegel, IEEE Trans. Microwave Theory Tech. 50, 910 (2002).

[2] J. H. Booske, Phys. Plasmas 15, 055502 (2008).

[3] S. Liu, C. Zhang, M. Hu, X. Chen, P. Zhang, S. Gong, T. Zhao, and R. Zhong, Appl. Phys. Lett. 104, 201104 (2014).

[4] V. L. Bratman, A. E. Fedotov, and P. B. Makhalov, Appl. Phys. Lett. 98, 061503 (2011).

[5] J. So, K. F. MacDonald, and N. I. Zheludev, Appl. Phys. Lett. 104, 201101 (2014).

[6] S. P. Mickan and X.-C. Zhang, Int. J. High Speed Electron. Syst. 13, 601 (2003).

[7] A. J. Fitzgerald, E. Berry, N. N. Zinov'ev, S. HomerVanniasinkam, R. E. Miles, J. M. Chamberlain, and M. A. Smith, J. Biol. Phys. 29, 123 (2004).

[8] V. S. Cherkassky, B. A. Knyazeva, V. V. Kubarev, G. N. Kulipanov, G. L. Kuryshev, A. N. Matveenko, A. K. Petrov, V. M. Popik, M. A. Scheglov, O. A. Shevchenko et al., Nucl. Instrum. Methods Phys. Res., Sect. A 543, 102 (2005).

[9] S. J. Papadakis, A. H. Monica, J. Yu, J. A. Miragliotta, R. Osiander, T. Antonsen, and G. Nusinovich, Proc. SPIE Int. Soc. Opt. Eng. 6549, 65490N (2007).

[10] P. Zhang, B. Hoff, Y. Y. Lau, D. M. French, and J. W. Luginsland, Phys. Plasmas 19, 123104 (2012).
[11] G. Adamo, K. F. MacDonald, Y. H. Fu, C. Wang, D. P. Tsai, F. J. G. de Abajo, and N. I. Zheludev, Phys. Rev. Lett. 103, 113901 (2009).

[12] S. J. Smith and E. M. Purcell, Phys. Rev. 92, 1069 (1953).

[13] P. M. van den Berg, J. Opt. Soc. Am. 63, 689 (1973).

[14] P. M. van den Berg and T. H. Tan, J. Opt. Soc. Am. 64, 325 (1974).

[15] J. Walsh, K. Woods, and S. Yeager, Nucl. Instrum. Methods Phys. Res., Sect. A 341, 277 (1994).

[16] A. Gover, P. Dvorkis, and U. Elisha, J. Opt. Soc. Am. B 1, 723 (1984).

[17] V. L. Bratman, N. S. Ginzburg, and M. I. Petelin, Opt. Commun. 30, 409 (1979).

[18] J. M. Wachtel, J. Appl. Phys. 50, 49 (1979).

[19] L. Schachter and A. Ron, Phys. Rev. A 40, 876 (1989).

[20] J. Urata, M. Goldstein, M. F. Kimmit, A. Naumov, C. Platt, and J. E. Walsh, Phys. Rev. Lett. 80, 516 (1998).

[21] K.-J. Kim and S.-B. Song, Nucl. Instrum. Methods Phys. Res., Sect. A 475, 158 (2001).

[22] H. L. Andrews and C. A. Brau, Phys. Rev. ST Accel. Beams 7, 070701 (2004).

[23] H. L. Andrews, C. H. Boulware, C. A. Brau, and J. D. Jarvis, in Proceedings of FEL2004 Conference (Comitato Conferenze Elettra, Trieste, Italy, 2004), pp. 278-281 (http://www.JACoW.org).

[24] H. L. Andrews, C. H. Boulware, C. A. Brau, and J. D. Jarvis, Phys. Rev. ST Accel. Beams 8, 050703 (2005).

[25] V. Kumar and K. J. Kim, Phys. Rev. E 73, 026501 (2006).

[26] K. J. Kim and V. Kumar, Phys. Rev. ST Accel. Beams 10, 080702 (2007).

[27] J. T. Donohue and J. Gardelle, Phys. Rev. ST Accel. Beams 8, 060702 (2005).

[28] J. T. Donohue and J. Gardelle, Phys. Rev. ST Accel. Beams 9, 060701 (2006).

[29] D. Li, K. Imasaki, Z. Yang, and G.-S. Park, Appl. Phys. Lett. 88, 201501 (2006).

[30] D. Li, K. Imasaki, Z. Yang, G.-S. Park, and S. Miyamoto, Jpn. J. Appl. Phys. 46, 601 (2007).

[31] Y.-M. Shin, J.-K. So, K.-H. Jang, and J.-H. Won, Appl. Phys. Lett. 90, 031502 (2007).

[32] Y.-M. Shin, J.-K. So, K.-H. Jang, J.-H. Won, A. Srivastava, and G.-S. Park, Phys. Rev. Lett. 99, 147402 (2007).

[33] D. Li, K. Imasaki, X. Gao, Z. Yang, and G.-S. Park, Appl. Phys. Lett. 91, 221506 (2007).

[34] D. Li, Z. Yang, K. Imasaki, and G. S. Park, Phys. Rev. ST Accel. Beams 9, 040701 (2006).

[35] K. L. Jensen, D. W. Feldman, N. A. Moody, and P. G. O'Shea, J. Appl. Phys. 99, 124905 (2006).

[36] A. Gover, Phys. Rev. ST Accel. Beams 8, 030701 (2005).

[37] A. Gover and E. Dyunin, Phys. Rev. ST Accel. Beams 8, 030702 (2005).

[38] S. E. Korby, A. S. Kesar, J. R. Sirigiri, and R. J. Temkin, Phys. Rev. Lett. 94, 054803 (2005).

[39] K. Mizuno and S. Ono, The Ledatron in "Infrared and Millimeter Waves", edited by K. J. Button (Academic Press, New York, 1979), Vol. 1, pp. 213-234.

[40] D. Wortman and R. Leavitt, The Orotron in "Infrared and Millimeter Waves", edited by K. J. Button (Academic Press, New York, 1983), Vol. 7, Chap. 7, pp. 322-376. 
[41] F. S. Rusin and G. D. Bogomolov, JETP Lett. 4, 236 (1966).

[42] G. S. Nusinovich, Phys. Plasmas 13, 053107 (2006).

[43] B. Goplen, L. Ludeking, D. Smithe, and G. Warren, Comput. Phys. Commun. 87, 54 (1995).

[44] Y. Y. Lau and D. Chernin, Phys. Fluids B 4, 3473 (1992).

[45] H. R. Johnson, Proc. IRE 43, 684 (1955).

[46] J. A. Swegle, Phys. Fluids 30, 1201 (1987).

[47] L. K. Ang and Y. Y. Lau, Phys. Plasmas 5, 4408 (1998).

[48] D. Kipnis, E. Dyunin, and A. Gover, in Proceedings of the 27th International Free Electron Laser Conference, JACoW/eConf C0508213 (2005), p. 328.
[49] D. Kipnis, Backward wave interaction and oscillation mechanisms in free electron lasers, Master's thesis, Tel Aviv University, 2007.

[50] A. S. Gilmour, Traveling Wave Tubes (Artech, Norwood, MA, 1994).

[51] V. Kumar and K.-J. Kim, in Proceedings of FEL 2006 (BESSY, Berlin, Germany, 2006), p. 67 (http://www .JACoW.org).

[52] A. Yariv, Optical Electronics (Oxford University Press, New York, 1990).

[53] T. Shintake et al., Nat. Photonics 2, 555 (2008). 